\title{
NON-COMMUNICATIVE FUNCTIONS CAN BE EQUALLY IMPORTANT FOR STUDIES OF LANGUAGE EVOLUTION
}

\author{
KOJI FUJITA \\ fujita.koji.3x@kyoto-u.ac.jp \\ Graduate School of Human and Environmental Studies, \\ Kyoto University, Kyoto, Japan
}

Studies of animal communication play a central role in today's evolutionary linguistics. I argue here that non-communicative behaviors of animals and humans may provide equally important insights into language evolution.

Contrary to the previously held view that language is totally unique to humans, we now understand (i) that language is a complex trait consisting of several subsystems each of which may have evolved independently in other species, and (ii) that language emerged as a result of a (re)combination of these subsystems (precursors). Among these subsystems is a hierarchical compositional syntax, which still enjoys a special status as the hallmark of human language to be found nowhere else in the animal kingdom. Syntax therefore is considered to be a perplexing barrier to the natural understanding of language evolution.

Fortunately, researchers are now beginning to overcome this barrier by carrying out experiments which purport to show that a rudimentary syntax exists in nonhuman animal communication systems. Most recently, Suzuki et al. (2016, 2017) claim that the Japanese tit (Parus minor) may have compositional syntax, by testing these birds in ingeniously devised experimental methods and showing that they respond distinctively to novel sequences of calls in accordance with call ordering.

However, a cursory review of their study reveals that there are still some deep gaps between human syntax and the alleged compositional syntax in birds (Fujita, 2017). Most notably, their experiments show at best that bird syntax is only narrowly based on linear order, whereas it is well attested that human 
language is dependent on hierarchy instead of (or in addition to) linearity. It is also unclear whether bird syntax has such typical structural properties of human syntax as recursiveness and endocentricity (headedness). Studies of primate alarm calls point to more or less a similar conclusion (Schlenker et al., 2016). In a nutshell, animal communication can be combinatorial but not compositional, as far as available evidence goes. As usual, absence of evidence is not evidence of absence, and further attempts to search for genuinely compositional syntax in nonhuman animals are highly welcome. In light of current situation, I make the following two points to make inquiries into language evolution even more integrative, constructive and fruitful.

(1) Animal communication should be better conceived of as a precursor to protolanguage with linear grammar, rather than to full human language with hierarchical grammar (Bickerton, 2014, Jackendoff \& Wittenberg, 2016). This allows one to focus on the shift from linear grammar to hierarchical grammar in understanding how human language emerged. Lack of true compositionality in animal communication hints at its similarity with exocentric compounds in human language (like birdbrain in its metonymic sense; Jackendoff, 2009, Progovac, 2015), but as I will argue there is still a remarkable difference in that exocentric compounds in human language involves endocentricity or semantic compositionality in nature.

(2) To explain the shift/expansion from linear grammar to hierarchical grammar, one needs to look beyond animal communication and investigate noncommunicative behaviors of other animals. Researchers disagree with respect to whether the original function of language was communication or not, but to say the least, given the non-monolithic nature of human language, it is unlikely that every subsystem of language evolved as an adaptation to communication from the beginning. In particular, the precursor to hierarchical grammar may be found in non-communicative functions like primitive tool use and tool making, shared by humans and nonhumans alike (Boeckx \& Fujita, 2014, but contra Berwick \& Chomsky, 2017).

This is plausible, for example, in light of the common neural substrates for syntax and action, and also the formal parallelism between syntactic structure building and hierarchical object manipulation. That communication may not be very relevant to the emergence of hierarchical grammar is also supported by the observation that hierarchical grammar is rather dysfunctional in terms of communicative efficiency (consider how even simple expressions like green tea cup or John said Mary laughed again can be structurally and semantically 
ambiguous, a communicative disadvantage which would be absent in linear grammar).

Healthy growth of evolutionary linguistics requires a much broader perspective which pays serious attention to both communicative and noncommunicative behaviors of humans and other animals alike.

\section{Acknowledgements}

This work has been supported by MEXT/JSPS Grant-in-Aid for Scientific Research on Innovative Areas \#4903 (Evolinguistics), JP17H06379, and JSPS Grant-in-Aid for Scientific Research (C) JP16K02765.

\section{References}

Berwick, R. C., \& Chomsky, N. (2017). Why only us: recent questions and answers. Journal of Neurolinguistics, 43B, 166-177.

Bickerton, D. (2014). More than nature needs: language, mind, and evolution. Cambridge, MA: Harvard University Press.

Boeckx, C., \& Fujita, K. (2014). Syntax, action, comparative cognitive science, and Darwinian thinking. Frontiers in Psychology, 5, 627.

Fujita, K. (2017). On the parallel evolution of syntax and lexicon: a Merge-only view. Journal of Neurolinguistics, 43B, 178-192.

Jackendoff, R. (2009). Compounding in the parallel architecture and conceptual semantics. In R. Lieber \& P. Štekauer (Eds.), The Oxford handbook of compounding (pp. 105-128). Oxford: Oxford University Press.

Jackendoff, R., \& Wittenberg, E. (2016). Linear grammar as a possible steppingstone in the evolution of language. Psychonomic Bulletin \& Review, 24, 219 224.

Progovac, L. (2014). Evolutionary syntax. Oxford: Oxford University Press.

Schlenker, P., Chemla, E., \& Zuberbühler, K. (2016). What do monkey calls mean? Trends in Cognitive Sciences, 20, 894-904.

Suzuki, T. N., Wheatcroft, D., \& Griesser, M. (2016). Experimental evidence for compositional syntax in bird calls. Nature Communications, 7,10986.

Suzuki, T. N., Wheatcroft, D., \& Griesser, M. (2017). Wild birds use an ordering rule to decode novel call sequences. Current Biology, 27, 23312336. 